\title{
Unkonventionelles zur Neuordnung der Delegierten Psychotherapie
}

\section{Urs Dudle}

Dr. med., Psychiater in Zürich

\author{
Die Neuordnung der kassenpflichtigen Psychotherapie wird zwischen Ärzten und \\ Psychologen kontrovers diskutiert. Hier ein Beitrag, welcher Altbewährtes und \\ Neues zusammenführt.
}

Seit knapp 25 Jahren arbeiten delegierte Psychologen in meiner Praxis. Im Gegensatz zur schlechten Presse, welche man uns gerne andichtet, arbeiten sie zu durchaus guten Konditionen. Ja, wir sind ein Team, machen auch regelmässig Intervision, wo wir schwierige Klienten diskutieren. Über die Diskussion der Neuordnung der Psychotherapie bin ich nicht sonderlich erfreut. Dies nicht aus dem Grunde, weil ich den Psychologen einen selbständigen Status verweigern möchte, sondern vor allem deshalb, weil man scheinbar das alte Modell neben dem neuen nicht weiterführen will und damit möglicherweise das Kind mit dem Bade ausschüttet.

Tatsächlich ist es so, dass viele Gründe für eine mögliche Selbständigkeit der Psychologen sprechen, es aber andererseits kaum echte Gründe gibt, warum Psychiater und Psychologen nicht weiterhin wie bis anhin bei der Delegierten Psychotherapie zusammenarbeiten sollen. Ganz im Gegenteil. Es gibt eine Menge Gründe, welche dafür sprechen.

- Das Modell der Delegierten Psychotherapie hat sich während Jahren gut bewährt und wird von Kliniken und Ambulatorien auch fraglos weitergeführt werden. Die aktuellen Engpässe in der Psychotherapie sind vor allem dem Mangel an Psychiatern geschuldet und damit auch dem Mangel an entsprechenden Stellenangeboten, was dann in seltenen Fällen zu schlechten Arbeitsbedingungen der angestellten Psychologen führen mag. Allerdings findet man in der Presse nicht selten völlig unrealistische Zahlen, was denn ein fairer Lohn sei. Je nach Kostenlage wird der Nettolohn eines Psychologen kaum viel mehr als etwa 52\% der abgerechneten Leistungen ausmachen können. Der Mangel an Psychiatern übrigens ist, wie wir wissen, unserer Gesundheitsund Bildungspolitik zu verdanken. Seit Jahren bildet die Schweiz zu wenige Ärzte aus, und die Psychiater sind trotz vieler kostspieliger Fortbildungsjahre die am schlechtesten verdienenden Ärzte. Und dies mit fallender Tendenz: So hat uns Psychiatern der neue TARMED von 2018 eine geschätzte Einkommensminderung von knapp 10\% beschert, und die offenbar vom BAG aktuell angedachten Massnahmen laufen erneut in dieselbe Richtung.

- Die Zusammenarbeit zwischen Psychiatern und Psychologen war und ist sowohl im stationären wie auch im ambulanten Setting sinnvoll. Den Psychologen fehlen immer wieder wichtige Elemente bei der Behandlung von komplexen Fällen. Dies betrifft insbesondere zusätzliche körperliche Leiden oder auch körperliche Ursachen von psychischen Störungen. Hinzu tritt natürlich die ganze Bandbreite von typischen psychosomatischen Erkrankungen. Bei schweren und akuten psychischen Störungen fehlen vielen Psychologen die nötige klinische Erfahrung und Sicherheit, und die Intervention oder wenigstens der Rat des Psychiaters ist in solchen Situationen dringend gefragt. Selbstverständlich ist in diesem Zusammenhang auch die Medikation zu nennen, welche noch immer Arztsache ist und insbesondere auch in akuten Situationen wichtig wird und öfters Hospitalisationen vermeiden kann. Hinzu kommen Krankheits- und andere Arztzeugnisse. Last, but not least sind Berichte und Gutachten zu nennen, bei welchen der Psychiater oft entscheidend mit eingebunden ist. Insbesondere verlangen viele Versicherungen, dass zwingend ein Facharzt den Bericht mit unterschreibt.

- Dieselbe Art von intensiver qualifizierter Zusammenarbeit kann natürlich von einem Anordnungsmodell nicht erreicht werden. Die meisten Mediziner ohne psychiatrischen Facharzttitel können die ihnen zugedachte Rolle logischerweise nicht kompetent übernehmen. Ein Anordnungsmodell nur durch Psychiater hat wohl ebenfalls seine Tücken, da viele Psychiater, wie ich selber auch, wohl wenig 
Lust auf eine Rolle haben, wo sie als blosse Patientendrehscheibe für Psychologen fungieren und sie vorwiegend für Anordnungen, Zeugnisse, Medikamente, Berichte und dergleichen zuständig sind.

- Allein schon die Tatsache der räumlichen Trennung zwischen dem anordnenden Arzt und dem Psychologen erschwert viele Abläufe erheblich und letztlich dürfte die faktische qualitativ hochstehende Zusammenarbeit zwischen Ärzten und Psychologen stark unter einem solchen neuen Modell leiden. Die enge Zusammenarbeit unter demselben Dach lässt sich nicht so einfach auslagern.

Beim neuen Modell sprechen manche etwa von 100 Millionen Mehrkosten. Realistischerweise muss man hier davon ausgehen, dass es bald zu einer Mengenausweitung kommen wird. In einem gewissen Ausmass ist etwa damit zu rechnen, dass alternative, bislang nur über die Zusatzversicherung abrechenbare Therapien (z.B. Reiki, Kinesiologie) unter dem Deckmantel der Psychotherapie abgerechnet werden, was in der Praxis kaum zu kontrollieren ist. Die Mehrkosten für das neue System dürften den veranschlagten Betrag innerhalb von fünf Jahren um das Einfache oder Mehrfache übersteigen. Alles andere halte ich für naiv oder für bewusste Augenwischerei. Kosten soll dieses zusätzliche Angebot andererseits so gut wie nichts, obwohl es eine Mengenzunahme der Psychotherapie zur Folge haben wird, was am Ende zwingend auf eine Mogelpackung hinauslaufen muss. Jetzt schon spricht die Politik von

CH-8001 Zürich

urs.dudle[at]gmx.net einer entsprechenden Tarifkorrektur bei Mengenausweitung. Dies könnte sich für die Psychologen selbst bald als Pferdefuss erweisen, und ihr Einkommen dürfte entgegen ihrer Hoffnung in Zukunft abnehmen. Hinzu kommen die verschärften Bedingungen bei den Kostengutsprachen für die Psychotherapien. Es droht hier, dass die Krankenkassen zunehmend restriktiv reagieren und laufende Therapien unterbrechen. Hinzu kommt die geäusserte Absicht, dass schon nach deutlich weniger Therapiestunden Berichte und Anträge geschrieben werden müssen. Letztlich läuft das dann wohl in die Richtung, wie wir es von Deutschland kennen, wo der administrative Aufwand im Verlaufe einer Psychotherapie eine Unmenge von Zeit verschlingt. Dass diese vielen Anträge dann oft nicht von psychiatrischen Fachärzten unterschrieben werden, zeigt, wie inkonsequent das angedachte System letztlich ist.

Aus diesen Gründen plädiere ich für eine Beibehaltung des Systems der Delegierten Psychotherapie. Dieses ist jedoch für Psychologen durch eine zweite, parallele, von der Grundversicherung gedeckte Psychotherapieform zu ergänzen. Das hat etwa den Vorteil, dass Psychologen nicht mehr zu schlechten Konditionen in der Praxis eines Psychiaters arbeiten müssen. (Sie werden es aber weiterhin während ihrer Ausbildung in den Kliniken tun!) Sollte es tatsächlich im hier beschriebenen Sinne zu zwei parallelen Systemen kommen, so muss dabei aber klar sein, dass bei einer raschen Mengenausweitung im neuen (Anordnungs-)System dieses selber und nicht die Delegierte Psychotherapie die Folgen davon tragen sollte. Ebenso gibt es keine Gründe, warum für die delegiert arbeitenden Psychologen nun plötzlich eine höhere Frequenz von Berichten oder Therapieanträgen gelten soll, hat sich doch für sie nichts geändert und arbeiten sie doch eng mit kompetenten Psychiatern zusammen und unterliegen somit einer stärkeren und kompetenteren Aufsicht als die freipraktizierenden Kollegen. Dass eine erhöhte Berichtfrequenz etwa gar noch für die Psychiater gelten sollte, wäre noch mehr aus der Luft gegriffen und für unseren Berufsstand völlig inakzeptabel. Es kann und darf nicht angehen, dass die Psychiater die negativen Folgen einer Mengenausweitung zu tragen haben, welche sie nicht verursacht haben und auch nicht wesentlich beeinflussen können.

Obwohl sich das System der Delegierten Psychotherapie über Jahrzehnte hinweg bewährt hat, ist in der aktuellen Diskussion kaum jemand auf die Idee gekommen, dieses System nicht einfach abzuschaffen, sondern es lediglich der Gerechtigkeit und der besseren Versorgung wegen durch ein zweites, paralleles Modell zu ergänzen. Genau dafür möchte ich eintreten, was im Übrigen auch so etwas wie einen (gut eidgenössischen) Kompromiss darstellt. 